\title{
The willingness for dietary and behavioral changes in frontline epidemic prevention workers after experiencing the outbreak of COVID-19 in China: a cross-sectional study
}

Weijun $\mathrm{Yu}^{1+}$, Ying $\mathrm{Xu}^{1 \dagger}$, Jianhua Zhang ${ }^{2}$, Qing Yuan ${ }^{1}$, Yanfang Guo ${ }^{1}$, Zhixue $\mathrm{Li}^{1}$, Xiangyang $\mathrm{He}^{1}$, Yan Ma ${ }^{1}$, Fengmin Cai ${ }^{1}$, Zheng Liu', Rencheng Zhao ${ }^{1}$, Dewang Wang ${ }^{1}$, Jialong Chen ${ }^{3^{*}}$ and Quanwei Guo ${ }^{2^{*}}$ (D)

\begin{abstract}
Background: The 2019 novel coronavirus disease (COVID-19) has had a massive impact on public health, resulting in sudden dietary and behavioral habit changes. Frontline epidemic prevention workers play a pivotal role against COVID-19. They must face high-risk infection conditions, insufficient anti-epidemic material supplies, mental pressure, and so on. COVID-19 seriously affects their dietary and behavioral habits, and poor habits make them more susceptible to COVID-19. However, their baseline dietary and behavioral habits before COVID-19 and their willingness to change these habits after the outbreak of COVID-19 remain unclear for these workers in China. This study aimed to explore the baseline dietary and behavioral habits of frontline workers and their willingness to change these habits after the outbreak of the epidemic; in addition, susceptible subgroups were identified by stratified analyses as targets of protective measures to keep them from being infected with COVID-19.
\end{abstract}

Methods: A cross-sectional study was conducted through an online questionnaire using a sample of 22,459 valid individuals living in China, including 9402 frontline epidemic prevention workers.

Results: Before COVID-19, 23.9\% of the frontline epidemic prevention workers reported a high-salt diet, $46.9 \%$ of them reported a high frequency of fried foods intake, and $50.9 \%$ of them smoked cigarettes. After the outbreak of COVID-19, 34.6\% of them expressed a willingness to reduce salt intake, and $43.7 \%$ of them wanted to reduce the frequency of pickled vegetables intake. A total of $37.9 \%$ of them expressed a willingness to decrease or quit smoking, and $44.5 \%$ of them wanted to increase sleep duration. Significant differences in the baseline dietary and behavioral habits and the willingness to change their habits were observed between frontline epidemic prevention workers and other participants.

Among the frontline epidemic prevention workers with poor dietary and behavioral habits before COVID-19, frontline epidemic prevention experience was a promoting factor for adopting worse dietary and behavioral habits, (Continued on next page)

\footnotetext{
* Correspondence: chenjialongaa@163.com; gqwsz@smu.edu.cn

tWeijun Yu and Ying Xu are co-first authors.

${ }^{3}$ School of Public Health, Guangdong Medical University, Dongguan 523808,

China

${ }^{2}$ Shenzhen Hospital, Southern Medical University, Shenzhen 518101, China

Full list of author information is available at the end of the article
}

(c) The Author(s). 2021 Open Access This article is licensed under a Creative Commons Attribution 4.0 International License, which permits use, sharing, adaptation, distribution and reproduction in any medium or format, as long as you give appropriate credit to the original author(s) and the source, provide a link to the Creative Commons licence, and indicate if changes were made. The images or other third party material in this article are included in the article's Creative Commons licence, unless indicated otherwise in a credit line to the material. If material is not included in the article's Creative Commons licence and your intended use is not permitted by statutory regulation or exceeds the permitted use, you will need to obtain permission directly from the copyright holder. To view a copy of this licence, visit http://creativecommons.org/licenses/by/4.0/. The Creative Commons Public Domain Dedication waiver (http://creativecommons.org/publicdomain/zero/1.0/) applies to the data made available in this article, unless otherwise stated in a credit line to the data. 


\begin{abstract}
(Continued from previous page)
including those in the high-salt intake subgroup $(\mathrm{OR}, 2.824 ; 95 \% \mathrm{Cl}, 2.341-3.405)$ and the 11-20 cigarettes/day subgroup (OR, 2.067; 95\% Cl, 1.359-3.143).

Conclusions: The dietary and behavioral habits of frontline epidemic prevention workers were worse than that those of other participants before COVID-19. They had a greater willingness to adopt healthy dietary and behavioral habits after experiencing the outbreak of COVID-19. However, frontline epidemic prevention workers with poor dietary and behavioral habits before COVID-19 continued in engage in these poor habits. Dietary and behavioral intervention policies should be drafted to protect their health, especially frontline epidemic prevention workers with poor habits at baseline.
\end{abstract}

Keywords: COVID-19, Frontline epidemic prevention workers, Dietary habits, Behavioral habits, Willingness to change, Cross-sectional study

\section{Background}

On January 30, 2020, the World Health Organization (WHO) Emergency Committee declared the 2019 novel coronavirus disease (COVID-19) a global health emergency [1]. It quickly spread worldwide and has become a public health emergency. As of December 31, 2020, the death toll of COVID-19 exceeded 1.7 million and the number of confirmed cases was more than 81 million worldwide [2]. COVID-19 has caused great harm to human health and the economy [3, 4], which has aroused widespread concern in society [5, 6]. Recent data suggested that only a few countries were able to prevent the spread of the epidemic and most countries are facing great challenges with COVID-19, especially in South Asia, South and North America, and Europe [7]. Moreover, some countries have suffered a second wave, and there may be more waves of coronavirus infection with the mutated coronavirus strains, with premature relaxation of interventions, with the economy restarting and with cool weather in the Northern Hemisphere [8, 9].

In this tough global fight against COVID-19, frontline epidemic prevention workers play a crucial role [10]. However, it has been reported that many frontline epidemic prevention workers have been infected and even died $[11,12]$. They must face large pressures with heavy workloads, high risk of infection, mental pressure, and depression and anxiety during the COVID-19 period [13-18]. Given the great influence of COVID-19, everyone had to change their dietary and behavioral habits to adapt to this current serious situation, including frontline epidemic prevention workers [19]. Willett et al. reported that unhealthy behaviors and lifestyles would lead to people suffering from low immunity and chronic diseases [20]. Studies have revealed that immunocompromised people and patients with chronic diseases have higher COVID-19 infection rates and mortality after infection [21-24]. However, the baseline dietary and behavioral habits of frontline epidemic prevention workers before COVID-19 and their willingness to change these habits after the outbreak of COVID-19 remain unclear in China. Therefore, research data are needed to develop evidence-driven strategies to keep frontline epidemic prevention workers healthy and to reduce their risk of being infected during the epidemic.

In this study, we investigated the baseline dietary and behavioral habits of frontline epidemic prevention workers before COVID-19 and further explored the relationship between the frontline epidemic prevention experience during the epidemic outbreak and their willingness to change dietary and behavioral habits after the outbreak of COVID-19 based on a cross-sectional study in China. To further explore targeted strategies, those who expressed a willingness to adopt worse dietary and behavioral habits were screened out by a stratified analysis. Our work will be useful for governments or organizations to develop interventions to protect frontline epidemic prevention workers from COVID-19, especially in subgroups with poor dietary and behavioral habits.

\section{Methods \\ Participants}

After the outbreak of COVID-19 in China, a crosssectional study was conducted online for all netizens on April 25, 2020. The participants were recruited by the snowball sampling method, and this nonprobability method had the advantages of convenience, high efficiency, and avoidance of direct contact during COVID19 [25]. The inclusion criteria were as follows: (1) they all volunteered to participate in this questionnaire survey, and (2) they independently completed the questionnaire without interference or logical errors. We defined frontline epidemic prevention workers as medical workers, community workers, policemen, volunteers, and others (anti-epidemic officers especially grassroots cadres, taxi drivers, delivery men, cleaners, construction workers of epidemic prevention facilities, workers who 
transfer people diagnosed or suspected of being infected with COVID-19, etc.). A total of 28,877 responders participated in the study in China.

\section{Data collection}

The online anonymous questionnaire, which permitted more authentic and reliable data than traditional paper questionnaires, was published in the WeChat public account (such as Twitter) of Bao'an District Hospital for Chronic Diseases Prevention and Cure. All participants could complete the online questionnaires by scanning a quick response code. Unified guidance language was used to introduce the study purposes and ensured data confidentiality to the participants. The system could intelligently prompt participants until the questionnaire was completed and submitted. Of the 28,877 responders, 1966 dropped out, 4452 were excluded for logical errors, and 22,459 were effective. A total of 9402 frontline epidemic prevention workers' valid questionnaires were collected, accounting for $41.9 \%$.

\section{Questionnaire}

A structured questionnaire (Table 1) with close-ended questions was developed after searching the literature [26] and consulting experts and included the following: (1) General characteristics: gender, age, height, weight, education level, marital status, occupation, main living place, and frontline epidemic prevention experience during the outbreak of COVID-19. (2) Baseline dietary habits before COVID-19: salt intake (high, medium, and low), intake frequency (days/week) of fried foods, sugary foods, pickled vegetables, processed meat products, fresh vegetables, fresh fruits, and protein-rich products. We defined sugary foods as sugar, sugary beverages, desserts, cookies, candies, fruit products, and dairy desserts and protein-rich products as meat, eggs, milk, and dairy. (3) The baseline behavioral habits before COVID-19: smoking (cigarettes/day), alcohol consumption frequency (days/week), sleep duration (hours/day), and physical exercise time (minutes or hours/week). (4) Willingness to change dietary habits after experiencing the outbreak of COVID-19: self-reported willingness to change (unchanged, increase, decrease, uncertain) dietary habits, including intake of salt, fried foods, sugary foods, pickled vegetables, processed meat products, fresh vegetables, fresh fruits, and protein-rich products after experiencing the outbreak of COVID-19. (5) Willingness to change behavioral habits after experiencing the outbreak of COVID-19: self-reported willingness to change behavioral habits, including smoking, alcohol consumption frequency, sleep duration, and physical exercise time after experiencing the outbreak of COVID-19.

\section{Statistical methods}

The original data were downloaded directly from the website. The SPSS 25.0 software package was used for statistical analysis. Measurement data (age and BMI) were analyzed by Student's $t$-tests. Categorical data were analyzed with chi-square tests. Salt intake (low $=1$, medium $=2$, and high $=3)$, intake frequency $(\leq$ 1 day $/$ week $=1,2-3$ days $/$ week $=2,4-6$ days $/$ week $=$ 3 , every day $=4$ ), smoking (no smoking $=1,1-5$ cigarettes $/$ day $=2,6-10$ cigarettes $/$ day $=3,11-20$ cigarettes $/$ day $=4,>20$ cigarettes $/$ day $=5$ ), sleep duration $(<5 \mathrm{~h} /$ day $=1,5-6 \mathrm{~h} /$ day $=2,6-7 \mathrm{~h} /$ day $=$ $3,7-8 \mathrm{~h} /$ day $=4,>8 \mathrm{~h} /$ day $=5)$, and physical exercise $(<30 \mathrm{~min} /$ week $=1,30-60 \mathrm{~min} /$ week $=2,1-2$ $\mathrm{h} /$ week $=3,2-3 \mathrm{~h} /$ week $=4,>3 \mathrm{~h} /$ week $=5$ ) were treated as ordinal variables. Gender, frontline epidemic prevention experience, and current residence place were treated as dichotomous variables. Education level, marital status, occupation, and willingness to change dietary and behavioral habits were regarded as nominal variables. The baseline dietary and behavioral habits were regarded as a stratification factor and those with unchanged willingness were regarded as a reference group in the multinomial logistic regression models. After adjusting for other characteristic factors (gender, age, BMI, education level, marital status, occupation, and current residence place), the willingness to change dietary and behavioral habits were investigated in frontline epidemic prevention workers after experiencing the outbreak of COVID19. For all analyses, the difference was statistically significant at $P<0.05$.

\section{Results}

\section{General information from the questionnaires}

From 10:00 on January 23, 2020 to 0:00 on April 8, 2020, Wuhan engaged in anti-epidemic warfare for these 76 days and then officially restarted; we defined this period as the outbreak of COVID-19 in China. To investigate frontline epidemic prevention workers' baseline dietary and behavioral habits before COVID19 , and their willingness to change dietary and behavioral habits after experiencing the outbreak of COVID-19, online questionnaires were conducted on April 25, 2020.

A total of 22,459 responders participated in this study, including 9402 (41.9\%) frontline epidemic prevention workers, and 13,057 (58.1\%) non-frontline epidemic prevention workers. Among them, 14,204 were males (63.2\%), and 8255 were females $(36.8 \%)$; $11,182(49.8 \%)$ were married, 10,567 (47.1\%) were unmarried, and $710(3.2 \%)$ were others (including remarried, cohabiting, separated, divorced, and widowed); and 20,650 (91.9\%) were in senior high 
Table 1 Investigated questions on baseline dietary and behavioral habits, and willingness to change dietary and behavioral habits after experiencing the outbreak of COVID-19

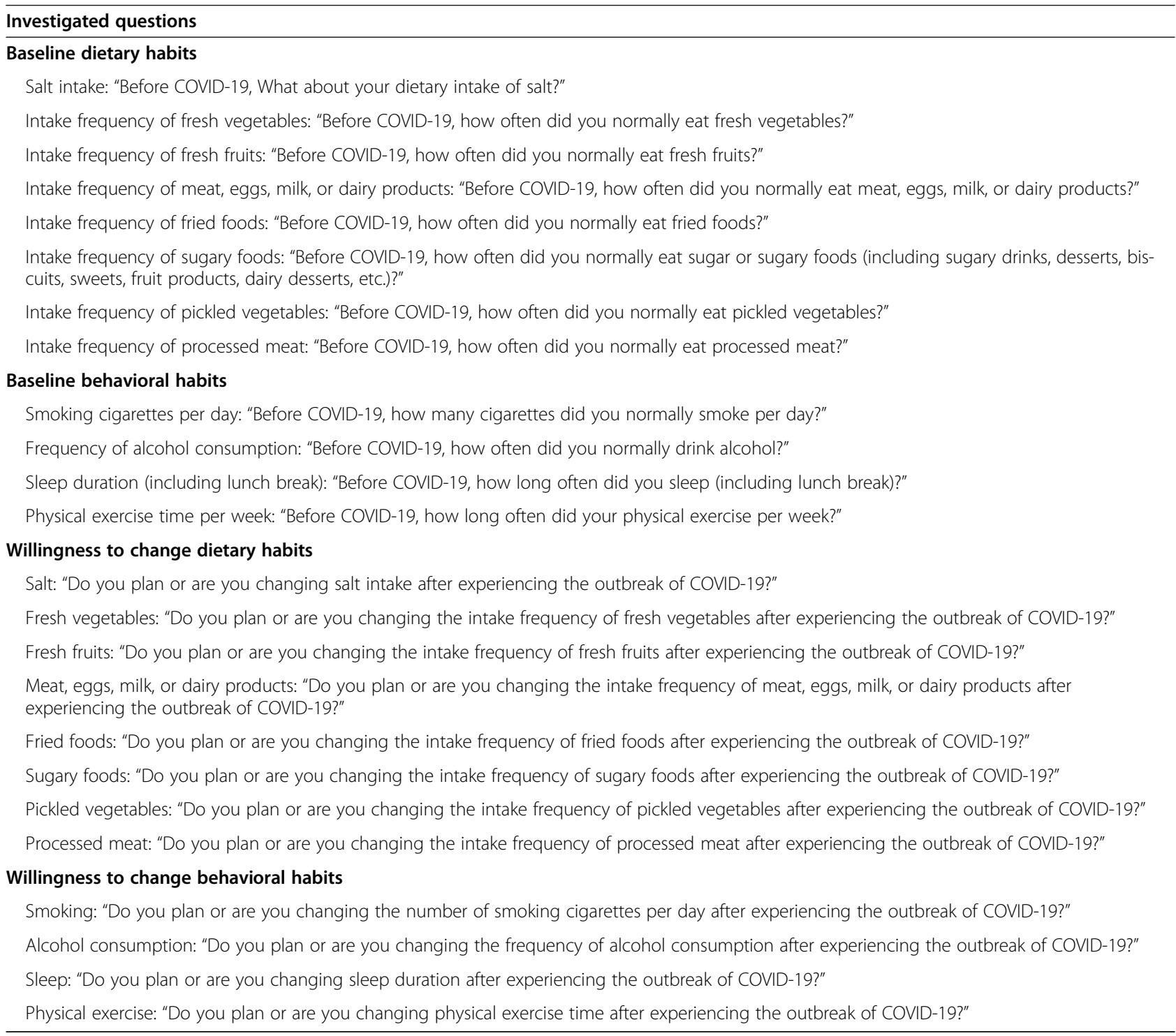

school and above, and 1809 (8.1\%) were in junior high school and below. The mean age and BMI were $27.88 \pm 7.84$ (years) and $22.05 \pm 4.88$, respectively. During the outbreak of COVID-19, 14,069 (62.6\%) lived in Guangdong Province, 292 (1.3\%) lived in Hubei, and 8098 (36.1\%) lived in other provinces (Table 2).

Information of the frontline epidemic prevention workers A total of 9402 frontline epidemic prevention workers' valid questionnaires were collected. Among them, 6392 were males $(68.0 \%)$ and 3010 were females (32.0\%); 5035 (53.6\%) were married, 4022 (42.8\%) were unmarried, and $345(3.7 \%)$ were others; $6336(67.4 \%)$ were in college and above and 3066 (32.6\%) in senior high school and below; $478(5.1 \%)$ were medical workers, 620 (6.6\%) were community workers, $124(1.3 \%)$ were policemen, and 8180 (87.0\%) were others. The mean age and BMI were $28.09 \pm$ 7.50 (years) and $21.93 \pm 4.85$, respectively. During the outbreak of COVID-19, 121 (1.3\%) lived in Hubei Province, and 9821 (98.7\%) lived in other provinces (Table 2).

\section{Univariate analysis of associations between frontline epidemic prevention experience and baseline dietary and behavioral habits before COVID-19}

Before COVID-19, 23.9\% of the frontline epidemic prevention workers reported that they had a high-salt diet, and $46.9 \%, 55.1 \%, 45.0 \%$, and $49.1 \%$ of them reported 
Table 2 Demographic characteristics of participants according to frontline epidemic prevention experience during the outbreak of COVID-19

\begin{tabular}{|c|c|c|c|c|c|}
\hline \multirow[t]{2}{*}{ Demographic characteristics } & \multicolumn{2}{|c|}{ Frontline epidemic prevention experience, $N(\%)$} & \multirow{2}{*}{$\begin{array}{l}\text { Total, } N= \\
22,459\end{array}$} & \multirow[t]{2}{*}{$T$ or $\mathrm{x}^{2}$} & \multirow{2}{*}{$\begin{array}{l}P \text { - } \\
\text { value }\end{array}$} \\
\hline & Yes, 9402 (41.9) & No, 13,057 (58.1) & & & \\
\hline \multicolumn{6}{|l|}{ Gender } \\
\hline Male & $6392(68.0)$ & $7812(59.8)$ & $14,204(63.2)$ & 156.399 & $<0.001$ \\
\hline Female & $3010(32.0)$ & $5245(40.2)$ & $8255(36.8)$ & & \\
\hline Age (years), mean $\pm S D$ & $28.09 \pm 7.50$ & $27.73 \pm 8.08$ & $27.88 \pm 7.84$ & -3.457 & 0.001 \\
\hline $\mathrm{BMI}$, mean $\pm \mathrm{SD}$ & $21.93 \pm 4.85$ & $22.13 \pm 4.91$ & $22.049 \pm 4.88$ & 3.058 & 0.002 \\
\hline \multicolumn{6}{|l|}{ Marital status } \\
\hline Married & $5035(53.6)$ & $6147(47.1)$ & $11,182(49.8)$ & 121.955 & $<0.001$ \\
\hline Never-married & $4022(42.8)$ & $6545(50.1)$ & $10,567(47.1)$ & & \\
\hline Others $^{a}$ & $345(3.7)$ & $365(2.8)$ & $710(3.2)$ & & \\
\hline \multicolumn{6}{|l|}{ Education } \\
\hline Primary schools and below & $172(1.8)$ & $164(1.3)$ & $336(1.5)$ & 38.409 & $<0.001$ \\
\hline Middle school & $602(6.4)$ & $871(6.7)$ & $1473(6.6)$ & & \\
\hline High school or technical secondary school & $2292(24.4)$ & $3582(27.4)$ & $5874(26.2)$ & & \\
\hline College & $2790(29.7)$ & $3717(28.5)$ & $6507(29.0)$ & & \\
\hline Bachelor's degree and above & $3546(37.7)$ & $4723(36.2)$ & $8269(36.8)$ & & \\
\hline \multicolumn{6}{|l|}{ Occupation } \\
\hline Medical workers & $478(5.1)$ & $233(1.8)$ & $711(3.2)$ & 421.452 & $<0.001$ \\
\hline Community worker & $620(6.6)$ & $391(3.0)$ & $1011(4.5)$ & & \\
\hline Policemen & $124(1.2)$ & $68(0.5)$ & $192(0.9)$ & & \\
\hline Other & $8180(87.0)$ & $12,365(94.7)$ & $20,545(91.5)$ & & \\
\hline \multicolumn{6}{|l|}{ Place of current residence } \\
\hline Hubei & $121(1.3)$ & $171(1.3)$ & $292(1.3)$ & 0.022 & 0.882 \\
\hline Other parts of China & $9281(98.7)$ & $12,886(98.7)$ & $22,167(98.7)$ & & \\
\hline
\end{tabular}

${ }^{a}$ Others included remarried, cohabiting, separated, divorced, and widowed

that their intake frequency of fried foods, sugary foods, pickled vegetables, and processed meat products, respectively, was 4-6 days/week or above. However, 21.6\%, $26.8 \%$, and $30.5 \%$ of them reported that their intake frequency of fresh vegetables, fresh fruits, and protein-rich products, respectively, was 2-3 days/week or below. Most of these data for the frontline epidemic prevention workers were higher than the data for the other participants, and these differences in dietary habits were statistically significant $(P<0.05)$ (Table 3$)$.

Of the frontline epidemic prevention workers, 50.9\% of them self-reported that they smoked cigarettes, $29.9 \%$ of them reported that their alcohol consumption frequency was 4-6 days/week or above, 59.5\% of them reported that their sleep duration was less than $7 \mathrm{~h} /$ day, and $54.1 \%$ of them reported that their physical exercise time was less than $1 \mathrm{~h}$ /week before COVID-19. Most of these data for the frontline epidemic prevention workers were higher than the data for other participants, and these differences in behavioral habits were statistically significant $(P<0.05)$ (Table 3$)$.
Univariate analysis of associations between frontline epidemic prevention experience and willingness to change dietary and behavioral habits after experiencing the outbreak of COVID-19

After experiencing the outbreak of COVID-19, 34.6\% of the frontline epidemic prevention workers reported their willingness to reduce their salt intake, and $45.2 \%, 40.7 \%$, $43.7 \%$, and $40.5 \%$ of them reported their willingness to reduce the frequency of their intake of fried foods, sugary foods, pickled vegetables, and processed meat products, respectively. Meanwhile, 52.1\%, 50.0\%, and $43.2 \%$ of them reported their willingness to increase the frequency of their intake of fresh vegetables, fresh fruits, and protein-rich products, respectively. Most of these data for the frontline epidemic prevention workers were higher than that the data for the other participants, and significant differences in willingness to change dietary habits were observed $(P<0.05)$ (Table 4$)$.

A total of $37.9 \%$ and $42.5 \%$ of the frontline epidemic prevention workers reported their willingness to decrease or quit their smoking and drinking alcohol 
Table 3 Univariate analysis of associations between frontline epidemic prevention experience and baseline dietary and behavioral habits before COVID-19

\begin{tabular}{|c|c|c|c|c|c|}
\hline \multirow[t]{2}{*}{ Baseline characteristics } & \multicolumn{2}{|c|}{ Frontline epidemic prevention experience, $N(\%)$} & \multirow{2}{*}{$\begin{array}{l}\text { Total, } N= \\
22,459\end{array}$} & \multirow[t]{2}{*}{$T$ or $x^{2}$} & \multirow[t]{2}{*}{$P$-value } \\
\hline & Yes, 9402 (41.9) & No, $13,057(58.1)$ & & & \\
\hline \multicolumn{6}{|l|}{ Baseline dietary habits } \\
\hline \multicolumn{6}{|l|}{ Salt intake } \\
\hline High & $2244(23.9)$ & $2610(20.0)$ & 4854 (21.6) & 50.693 & $<0.001$ \\
\hline Medium & $5767(61.3)$ & $8322(63.7)$ & $14,089(62.7)$ & & \\
\hline Low & $1391(14.8)$ & $2125(16.3)$ & $3516(15.7)$ & & \\
\hline \multicolumn{6}{|c|}{ Intake frequency of fresh vegetables } \\
\hline Every day & $3987(42.4)$ & $6561(50.2)$ & $10,548(47.0)$ & 153.388 & $<0.001$ \\
\hline 4-6 days/week & $3382(36.0)$ & $3810(29.2)$ & $7192(32.0)$ & & \\
\hline 2-3 days/week & $1791(19.0)$ & $2355(18.0)$ & $4146(18.5)$ & & \\
\hline$\leq 1$ day/week & $242(2.6)$ & $331(2.5)$ & $573(2.6)$ & & \\
\hline \multicolumn{6}{|c|}{ Intake frequency of fresh fruits } \\
\hline Every day & $3510(37.3)$ & $5233(40.1)$ & $8743(38.9)$ & 107.917 & $<0.001$ \\
\hline 4-6 days/week & 3379 (35.9) & $3903(29.9)$ & $7282(32.4)$ & & \\
\hline 2-3 days/week & $2177(23.2)$ & $3255(24.9)$ & $5432(24.2)$ & & \\
\hline$\leq 1$ day/week & $336(3.6)$ & $666(5.1)$ & $1002(4.5)$ & & \\
\hline \multicolumn{6}{|c|}{ Intake frequency of meat, eggs, milk, or dairy products } \\
\hline Every day & $3113(33.1)$ & 4779 (36.6) & $7892(35.1)$ & 87.739 & $<0.001$ \\
\hline 4-6 days/week & $3430(36.5)$ & $4041(30.9)$ & $7471(33.3)$ & & \\
\hline 2-3 days/week & $2459(26.2)$ & $3518(26.9)$ & $5977(26.6)$ & & \\
\hline$\leq 1$ day/week & $400(4.3)$ & $719(5.5)$ & $1119(5.0)$ & & \\
\hline \multicolumn{6}{|c|}{ Intake frequency of fried foods } \\
\hline Every day & $1417(15.1)$ & $1574(12.1)$ & $2991(13.3)$ & 372.657 & $<0.001$ \\
\hline 4-6 days/week & $2991(31.8)$ & $3183(24.4)$ & $6174(27.5)$ & & \\
\hline 2-3 days/week & 2995 (31.9) & 4135 (31.7) & $7130(31.7)$ & & \\
\hline$\leq 1$ day/week & 1999 (21.3) & 4165 (31.9) & $6164(27.4)$ & & \\
\hline \multicolumn{6}{|c|}{ Intake frequency of sugary foods } \\
\hline Every day & 2032 (21.6) & $2643(20.2)$ & $4675(20.8)$ & 195.740 & $<0.001$ \\
\hline 4-6 days/week & $3150(33.5)$ & $3569(27.3)$ & $6719(29.9)$ & & \\
\hline 2-3 days/week & $3144(33.4)$ & $4625(35.4)$ & 7769 (34.6) & & \\
\hline$\leq 1$ day/week & $1076(11.4)$ & $2220(17.0)$ & $3296(14.7)$ & & \\
\hline \multicolumn{6}{|c|}{ Intake frequency of pickled vegetables } \\
\hline Every day & $1426(15.2)$ & $1726(13.2)$ & $3152(14.0)$ & 419.729 & $<0.001$ \\
\hline 4-6 days/week & $2801(29.8)$ & $2972(22.8)$ & $5773(25.7)$ & & \\
\hline 2-3 days/week & 3179 (33.8) & $4007(30.7)$ & $7186(32.0)$ & & \\
\hline$\leq 1$ day/week & $1996(21.2)$ & $4352(33.3)$ & $6348(28.3)$ & & \\
\hline \multicolumn{6}{|c|}{ Intake frequency of processed meat products } \\
\hline Every day & $1548(16.5)$ & $1848(14.2)$ & $3396(15.1)$ & 380.866 & $<0.001$ \\
\hline 4-6 days/week & 3065 (32.6) & $3240(24.8)$ & $6305(28.1)$ & & \\
\hline 2-3 days/week & $3210(34.1)$ & $4465(34.2)$ & $7675(34.2)$ & & \\
\hline$\leq 1$ day/week & $1579(16.8)$ & $3504(26.8)$ & $5083(22.6)$ & & \\
\hline
\end{tabular}

\section{Baseline behavioral habits}


Table 3 Univariate analysis of associations between frontline epidemic prevention experience and baseline dietary and behavioral habits before COVID-19 (Continued)

\begin{tabular}{|c|c|c|c|c|c|}
\hline \multirow[t]{2}{*}{ Baseline characteristics } & \multicolumn{2}{|c|}{ Frontline epidemic prevention experience, $N(\%)$} & \multirow{2}{*}{$\begin{array}{l}\text { Total, } N= \\
22,459\end{array}$} & \multirow[t]{2}{*}{$T$ or $x^{2}$} & \multirow[t]{2}{*}{$P$-value } \\
\hline & Yes, 9402 (41.9) & No, $13,057(58.1)$ & & & \\
\hline$>20$ cigarettes per day & $148(1.6)$ & $135(1.0)$ & $283(1.3)$ & 721.002 & $<0.001$ \\
\hline 11-20 cigarettes per day & $694(7.4)$ & $727(5.6)$ & $1421(6.3)$ & & \\
\hline 6-10 cigarettes per day & $1971(21.0)$ & $1732(13.3)$ & $3703(16.5)$ & & \\
\hline $1-5$ cigarettes per day & $1963(20.9)$ & $1726(13.2)$ & $3689(16.4)$ & & \\
\hline No smoking & $4626(49.2)$ & $8737(66.9)$ & $13,363(59.5)$ & & \\
\hline \multicolumn{6}{|l|}{ Alcohol consumption/week } \\
\hline Every day & $992(10.6)$ & $1017(7.8)$ & $2009(8.9)$ & 1024.661 & $<0.001$ \\
\hline 4-6 days/week & $1813(19.3)$ & $1386(10.6)$ & $3199(14.2)$ & & \\
\hline 2-3 days/week & 2309 (24.6) & $2214(17.0)$ & $4523(20.1)$ & & \\
\hline$\leq 1$ day/week & $1537(16.3)$ & $2021(15.5)$ & $3558(15.8)$ & & \\
\hline No drinking & $2751(29.3)$ & $6419(49.2)$ & $9170(40.8)$ & & \\
\hline \multicolumn{6}{|c|}{ Sleep duration/day (including lunch break) } \\
\hline$<5$ h/day & $800(8.5)$ & $777(6.0)$ & $1577(7.0)$ & 231.381 & $<0.001$ \\
\hline $5-6$ h/day & $1799(19.1)$ & $1867(14.3)$ & $3666(16.3)$ & & \\
\hline 6-7 h/day & $3000(31.9)$ & $3986(30.5)$ & $6986(31.1)$ & & \\
\hline $7-8$ h/day & $2851(30.3)$ & $4668(35.8)$ & 7519 (33.5) & & \\
\hline$>8$ h/day & $952(10.1)$ & 1759 (13.5) & $2711(12.1)$ & & \\
\hline \multicolumn{6}{|l|}{ Physical exercise time/week } \\
\hline$<30 \mathrm{~min} /$ week & $1682(17.9)$ & $3772(28.9)$ & $5454(24.3)$ & 424.613 & $<0.001$ \\
\hline 30 min-1 h/week & $3401(36.2)$ & $4614(35.3)$ & $8015(35.7)$ & & \\
\hline $1-2 \mathrm{~h} /$ week & $2752(29.3)$ & $2907(22.3)$ & $5659(25.2)$ & & \\
\hline 2-3 h/week & $1081(11.5)$ & $1136(8.7)$ & $2217(9.9)$ & & \\
\hline$>3 \mathrm{~h} /$ week & $486(5.2)$ & $628(4.8)$ & $1114(5.0)$ & & \\
\hline
\end{tabular}

consumption, and $44.5 \%$ and $53.7 \%$ of them had a willingness to increase sleep duration and physical exercise time, respectively. All these data for the frontline epidemic prevention workers were higher than the data for other participants, and significant differences in willingness to change behavioral habits between them were observed $(P<0.05)$ (Table 4$)$.

\section{Multivariable analysis of associations between frontline epidemic prevention experience and willingness to change dietary habits}

With baseline dietary habit as a stratification factor and unwillingness to change dietary habit as a reference group, and after adjustments were made for gender, age, BMI, education level, marital status, occupation, and main living place, the stratified analysis of the multinomial logistic regression models showed that the frontline epidemic prevention workers with bad dietary habits were more likely to choose worse dietary habits after experiencing the outbreak of COVID-19. Regarding salt intake, frontline epidemic prevention workers with high-salt intake were more likely to increase salt intake than other participants (OR, 2.824; 95\% CI, 2.341-3.405). Regarding those with the lowest level frequency of fresh fruits ( $\leq 1$ day/week), frontline epidemic prevention workers expressed a willingness to decrease the intake frequency of fresh fruits (OR, 3.000; 95\% CI, 1.922-4.682). Moreover, regarding those with the highest-level frequency (every day/week) of sugary foods and fried foods, frontline epidemic prevention workers expressed a willingness to increase their sugary foods intake (OR, 1.683; 95\% CI, 1.424-1.988) and fried foods intake (OR, 1.566; 95\% CI, 1.271-1.931). Furthermore, the frontline epidemic prevention workers had other kinds of poor dietary habits, including the highest frequency intake of pickled vegetables and processed meat products and the lowest frequency intake of fresh vegetables, and protein-rich products (Table 5). 
Table 4 Univariate analysis of associations between frontline epidemic prevention experience and willingness to change dietary and behavioral habits after experiencing the outbreak of COVID-19

\begin{tabular}{|c|c|c|c|c|c|}
\hline \multirow{2}{*}{$\begin{array}{l}\text { Variables } \\
\text { characteristics }\end{array}$} & \multicolumn{2}{|c|}{ Frontline epidemic prevention experience, $N(\%)$} & \multirow{2}{*}{$\begin{array}{l}\text { Total, } \\
n=22,459\end{array}$} & \multirow[t]{2}{*}{$T$ or $x^{2}$} & \multirow[t]{2}{*}{$P$-value } \\
\hline & Yes, 9402 (41.9) & No, 13,057 (58.1) & & & \\
\hline \multicolumn{6}{|c|}{ Willingness to change dietary habits } \\
\hline \multicolumn{6}{|l|}{ Salt intake } \\
\hline Unchange & $3741(39.8)$ & $6683(51.2)$ & $10,424(46.4)$ & 582.069 & $<0.001$ \\
\hline Increase & $2000(21.3)$ & $1536(11.8)$ & $3536(15.7)$ & & \\
\hline Decrease & $3257(34.6)$ & $3900(29.9)$ & $7157(31.9)$ & & \\
\hline Uncertain & $404(4.3)$ & $938(7.2)$ & $1342(6.0)$ & & \\
\hline \multicolumn{6}{|c|}{ Intake frequency of fresh vegetables } \\
\hline Unchange & $2892(29.8)$ & $5202(39.8)$ & 8004 (35.6) & 310.811 & $<0.001$ \\
\hline Increase & $4896(52.1)$ & $5774(44.2)$ & $10,670(47.5)$ & & \\
\hline Decrease & $1399(14.9)$ & $1467(11.2)$ & $2866(12.8)$ & & \\
\hline Uncertain & $305(3.2)$ & $614(4.7)$ & $919(4.1)$ & & \\
\hline \multicolumn{6}{|c|}{ Intake frequency of fresh fruits } \\
\hline Unchange & $2811(29.9)$ & $5059(38.7)$ & $7870(35.0)$ & 304.083 & $<0.001$ \\
\hline Increase & $4698(50.0)$ & $5786(44.3)$ & $10,484(46.7)$ & & \\
\hline Decrease & $1594(11.9)$ & $1553(11.9)$ & $3147(14.0)$ & & \\
\hline Uncertain & $299(3.2)$ & $659(5.0)$ & $958(4.3)$ & & \\
\hline \multicolumn{6}{|c|}{ Intake frequency of meat, eggs, milk, or dairy products } \\
\hline Unchange & $3059(32.5)$ & $5697(43.6)$ & $8756(39.0)$ & 365.679 & $<0.001$ \\
\hline Increase & $4063(43.2)$ & $4499(34.5)$ & $8562(38.1)$ & & \\
\hline Decrease & $1920(20.4)$ & $2137(16.4)$ & $4057(18.1)$ & & \\
\hline Uncertain & $360(3.8)$ & $724(5.5)$ & $1084(4.8)$ & & \\
\hline \multicolumn{6}{|c|}{ Intake frequency of fried foods } \\
\hline Unchange & $2344(24.9)$ & $4253(32.6)$ & $6597(29.4)$ & 486.665 & $<0.001$ \\
\hline Increase & $2437(25.9)$ & $1957(15.0)$ & $4394(19.6)$ & & \\
\hline Decrease & $4254(45.2)$ & $6080(46.6)$ & $10,334(46.0)$ & & \\
\hline Uncertain & $367(3.9)$ & $767(5.9)$ & $1134(5.0)$ & & \\
\hline \multicolumn{6}{|c|}{ Intake frequency of sugary foods } \\
\hline Unchange & $2523(26.8)$ & $4654(35.6)$ & $7177(32.0)$ & 552.086 & $<0.001$ \\
\hline Increase & $2617(27.8)$ & $2091(16.0)$ & $4708(21.0)$ & & \\
\hline Decrease & $3822(40.7)$ & $5378(41.2)$ & $9200(41.0)$ & & \\
\hline Uncertain & $440(4.7)$ & $934(7.2)$ & $1374(6.1)$ & & \\
\hline \multicolumn{6}{|c|}{ Intake frequency of pickled vegetables } \\
\hline Unchange & $2375(25.3)$ & $4411(33.8)$ & $6786(30.2)$ & 462.560 & $<0.001$ \\
\hline Increase & $2437(25.9)$ & $2062(15.8)$ & $4499(20.0)$ & & \\
\hline Decrease & $4113(43.7)$ & $5602(42.9)$ & $9715(43.3)$ & & \\
\hline Uncertain & $477(5.1)$ & $982(7.5)$ & $1459(6.5)$ & & \\
\hline \multicolumn{6}{|c|}{ Intake frequency of processed meat products } \\
\hline Unchange & $2548(27.1)$ & $4495(34.4)$ & $7043(31.4)$ & 413.992 & $<0.001$ \\
\hline Increase & $2573(27.4)$ & $2246(17.2)$ & $4819(21.5)$ & & \\
\hline Decrease & $3809(40.5)$ & $5319(40.7)$ & $9128(40.6)$ & & \\
\hline Uncertain & $472(5.0)$ & 997 (7.6) & $1469(6.5)$ & & \\
\hline
\end{tabular}


Table 4 Univariate analysis of associations between frontline epidemic prevention experience and willingness to change dietary and behavioral habits after experiencing the outbreak of COVID-19 (Continued)

\begin{tabular}{|c|c|c|c|c|c|}
\hline \multirow{2}{*}{$\begin{array}{l}\text { Variables } \\
\text { characteristics }\end{array}$} & \multicolumn{2}{|c|}{ Frontline epidemic prevention experience, $N(\%)$} & \multirow{2}{*}{$\begin{array}{l}\text { Total, } \\
n=22,459\end{array}$} & \multirow[t]{2}{*}{$T$ or $x^{2}$} & \multirow[t]{2}{*}{$P$-value } \\
\hline & Yes, $9402(41.9)$ & No, 13,057 (58.1) & & & \\
\hline \multicolumn{6}{|c|}{ Willingness to change behavioral habits } \\
\hline \multicolumn{6}{|c|}{ Smoking cigarettes/day } \\
\hline Unchange & $4074(43.3)$ & $7867(60.3)$ & $11,941(53.2)$ & 774.274 & $<0.001$ \\
\hline Increase & $1565(16.6)$ & $1111(8.5)$ & $2676(11.9)$ & & \\
\hline Decrease & $2400(25.5)$ & $2362(18.1)$ & $4762(21.2)$ & & \\
\hline Quit smoking & $1163(12.4)$ & $1341(10.3)$ & $2504(11.1)$ & & \\
\hline Uncertain & $200(2.1)$ & $376(2.9)$ & $576(2.6)$ & & \\
\hline \multicolumn{6}{|c|}{ Alcohol consumption/week } \\
\hline Unchange & $3570(38.0)$ & $6998(53.6)$ & $10,568(47.1)$ & 720.118 & $<0.001$ \\
\hline Increase & $1552(16.5)$ & $1173(9.0)$ & $2725(12.1)$ & & \\
\hline Decrease & $2815(29.9)$ & $2891(22.1)$ & $5706(25.4)$ & & \\
\hline Quit drinking & $1184(12.6)$ & $1401(10.7)$ & 2585 (11.5) & & \\
\hline Uncertain & $281(3.0)$ & $594(4.5)$ & $875(3.9)$ & & \\
\hline \multicolumn{6}{|c|}{ Sleep duration/day } \\
\hline Unchange & $2793(29.7)$ & $5272(40.4)$ & 8065 (35.9) & 349.024 & $<0.001$ \\
\hline Increase & $4181(44.5)$ & $5029(38.5)$ & $9210(41.0)$ & & \\
\hline Decrease & $1964(20.9)$ & $1945(14.9)$ & 3909 (17.4) & & \\
\hline Uncertain & $464(4.9)$ & $811(6.2)$ & $1275(5.7)$ & & \\
\hline \multicolumn{6}{|c|}{ Physical exercise time/week } \\
\hline Unchange & $2236(23.8)$ & 3765 (28.8) & $6001(26.7)$ & 173.142 & $<0.001$ \\
\hline Increase & $5387(57.3)$ & $7284(55.8)$ & $12,671(56.4)$ & & \\
\hline Decrease & $1516(16.1)$ & 1479 (11.3) & 2995 (13.3) & & \\
\hline Uncertain & $263(2.8)$ & $529(4.1)$ & $792(3.5)$ & & \\
\hline
\end{tabular}

Multivariable analysis of associations between frontline epidemic prevention experience and willingness to change behavioral habits

Using the same method as described in the "Multivariable analysis of associations between frontline epidemic prevention experience and willingness to change dietary habits" section, the data showed that frontline epidemic prevention workers who smoked 1-10 cigarettes/day were not willing to quit smoking but would increase the number of more cigarettes smoked. Regarding those with the highest frequency of alcohol consumption (every day/week), the frontline epidemic prevention experience showed a positive correlation with a willingness to increase the frequency of consuming alcohol (OR, 1.422; 95\% CI, 1.085-1.865). The frontline epidemic prevention workers with 5-6 h of sleep duration expressed a willingness to decrease their sleep time (OR, 1.634; 95\% CI, 1.301-2.053). The frontline epidemic prevention workers who engaged in physical exercise less than 30 min/week, expressed a willingness to decrease their physical exercise time (OR, 1.379; 95\% CI, 1.041-1.828). Our data suggested that some of the frontline epidemic prevention workers expressed a willingness to adopt worse behavioral habits after experiencing the outbreak of COVID-19, especially among those in poor habits subgroups (Table 6).

\section{Discussion}

COVID-19 has quickly spread worldwide since the outbreak in Wuhan. Zhou et al. [27] reported that health care workers are more susceptible to COVID-19. Many frontline epidemic prevention workers have been infected by coronavirus at work in almost in every country, and some of them have died in the fight against the epidemic [28]. Frontline epidemic prevention workers are the most valuable resource of every country, and the Lancet has suggested the government protect health care workers [12].

Frontline epidemic prevention workers are suffering from heavy workloads, high risk of infection, discrimination from other citizens, and so on. Most of them have experienced mental pressure, depression, and anxiety during the outbreak of COVID-19, especially those who have worked in seriously affected areas [10, 13, 29, 30]. 
Table 5 Multivariate analysis of associations between frontline epidemic prevention experience and willingness to change dietary habits after experiencing the outbreak of COVID-19

\begin{tabular}{|c|c|c|c|c|}
\hline Intake frequency of dietary habits ${ }^{a}$ & Willingness $^{\mathbf{b}}$ & OR & OR $(95 \% \mathrm{Cl})$ & $P$-value \\
\hline \multicolumn{5}{|l|}{ Salt intake } \\
\hline \multirow[t]{3}{*}{ High } & Increase & 2.824 & $2.341-3.405$ & $<0.001$ \\
\hline & Decrease & 1.096 & $0.957-1.254$ & 0.184 \\
\hline & Uncertain & 0.512 & $0.369-0.709$ & $<0.001$ \\
\hline \multirow[t]{3}{*}{ Medium } & Increase & 2.047 & $1.859-2.255$ & $<0.001$ \\
\hline & Decrease & 1.538 & $1.418-1.669$ & $<0.001$ \\
\hline & Uncertain & 0.800 & $0.685-0.934$ & 0.005 \\
\hline \multirow[t]{3}{*}{ Low } & Increase & 1.544 & $1.210-1.969$ & 0.001 \\
\hline & Decrease & 1.737 & $1.480-2.039$ & $<0.001$ \\
\hline & Uncertain & 1.204 & $0.893-1.622$ & 0.223 \\
\hline \multirow[t]{3}{*}{ Total } & Increase & 2.114 & $1.952-2.289$ & $<0.001$ \\
\hline & Decrease & 1.448 & $1.359-1.542$ & $<0.001$ \\
\hline & Uncertain & 0.789 & $0.696-0.895$ & $<0.001$ \\
\hline \multicolumn{5}{|l|}{ Intake frequency of fresh vegetables } \\
\hline \multirow[t]{3}{*}{ Every day } & Increase & 1.400 & $1.285-1.525$ & $<0.001$ \\
\hline & Decrease & 1.407 & $1.166-1.698$ & $<0.001$ \\
\hline & Uncertain & 0.698 & $0.540-0.901$ & 0.006 \\
\hline \multirow[t]{3}{*}{ 4-6 days/week } & Increase & 1.650 & $1.456-1.869$ & $<0.001$ \\
\hline & Decrease & 1.693 & $1.442-1.988$ & $<0.001$ \\
\hline & Uncertain & 1.104 & $0.814-1.497$ & 0.525 \\
\hline \multirow[t]{3}{*}{ 2-3 days/week } & Increase & 1.385 & $1.146-1.673$ & 0.001 \\
\hline & Decrease & 1.489 & $1.207-1.837$ & $<0.001$ \\
\hline & Uncertain & 0.975 & $0.715-1.329$ & 0.872 \\
\hline \multirow[t]{3}{*}{$\leq 1$ day/week } & Increase & 1.661 & $1.009-2.734$ & 0.046 \\
\hline & Decrease & 1.659 & $0.988-2.787$ & 0.055 \\
\hline & Uncertain & 1.041 & $0.587-1.844$ & 0.891 \\
\hline \multirow[t]{3}{*}{ Total } & Increase & 1.526 & $1.436-1.622$ & $<0.001$ \\
\hline & Decrease & 1.584 & $1.450-1.731$ & $<0.001$ \\
\hline & Uncertain & 0.907 & $0.782-1.053$ & 0.199 \\
\hline \multicolumn{5}{|l|}{ Intake frequency of fresh fruits } \\
\hline \multirow[t]{3}{*}{ Every day } & Increase & 1.359 & $1.237-1.493$ & $<0.001$ \\
\hline & Decrease & 1.696 & $1.384-2.079$ & $<0.001$ \\
\hline & Uncertain & 0.693 & $0.492-0.977$ & 0.036 \\
\hline \multirow[t]{3}{*}{ 4-6 days/week } & Increase & 1.599 & $1.410-1.813$ & $<0.001$ \\
\hline & Decrease & 1.986 & $1.696-2.327$ & $<0.001$ \\
\hline & Uncertain & 1.001 & $0.750-1.337$ & 0.994 \\
\hline \multirow[t]{3}{*}{ 2-3 days/week } & Increase & 1.495 & $1.279-1.748$ & $<0.001$ \\
\hline & Decrease & 1.806 & $1.514-2.155$ & $<0.001$ \\
\hline & Uncertain & 0.983 & $0.747-1.295$ & 0.905 \\
\hline \multirow[t]{3}{*}{$\leq 1$ day/week } & Increase & 1.794 & $1.213-2.653$ & 0.003 \\
\hline & Decrease & 3.000 & $1.922-4.682$ & $<0.001$ \\
\hline & Uncertain & 1.343 & $0.858-2.103$ & 0.197 \\
\hline Total & Increase & 1.412 & $1.328-1.501$ & $<0.001$ \\
\hline
\end{tabular}


Table 5 Multivariate analysis of associations between frontline epidemic prevention experience and willingness to change dietary habits after experiencing the outbreak of COVID-19 (Continued)

\begin{tabular}{lllll}
\hline Intake frequency of dietary habits $^{\mathbf{a}}$ & Willingness $^{\mathbf{b}}$ & OR & OR (95\% Cl) & $\boldsymbol{P}_{\text {-value }}$ \\
\hline & Decrease & 1.694 & $1.555-1.846$ & 0.001 \\
& Uncertain & 0.820 & $0.708-0.950$
\end{tabular}

Intake frequency of meat, eggs, milk, or dairy products

\begin{tabular}{|c|c|c|c|c|}
\hline \multirow[t]{3}{*}{ Every day } & Increase & 1.696 & $1.521-1.891$ & $<0.001$ \\
\hline & Decrease & 1.242 & $1.038-1.485$ & 0.018 \\
\hline & Uncertain & 0.747 & $0.539-1.036$ & 0.080 \\
\hline \multirow[t]{3}{*}{ 4-6 days/week } & Increase & 1.892 & $1.682-2.127$ & $<0.001$ \\
\hline & Decrease & 1.717 & $1.491-1.977$ & $<0.001$ \\
\hline & Uncertain & 1.011 & $0.771-1.3273$ & 0.935 \\
\hline \multirow[t]{3}{*}{ 2-3 days/week } & Increase & 1.468 & $1.277-1.688$ & $<0.001$ \\
\hline & Decrease & 1.733 & $1.490-2.016$ & $<0.001$ \\
\hline & Uncertain & 1.042 & $0.820-1.325$ & 0.737 \\
\hline \multirow[t]{3}{*}{$\leq 1$ day/week } & Increase & 1.252 & $0.877-1.786$ & 0.216 \\
\hline & Decrease & 1.862 & $1.291-2.685$ & 0.001 \\
\hline & Uncertain & 1.232 & $0.828-1.834$ & 0.303 \\
\hline \multirow[t]{3}{*}{ Total } & Increase & 1.615 & $1.518-1.719$ & $<0.001$ \\
\hline & Decrease & 1.533 & $1.437-1.678$ & $<0.001$ \\
\hline & Uncertain & 0.923 & $0.805-1.059$ & 0.254 \\
\hline \multicolumn{5}{|c|}{ Intake frequency of fried foods } \\
\hline \multirow[t]{3}{*}{ Every day } & Increase & 1.566 & $1.271-1.931$ & $<0.001$ \\
\hline & Decrease & 1.152 & $0.949-1.399$ & 0.153 \\
\hline & Uncertain & 0.777 & $0.460-1.314$ & 0.347 \\
\hline \multirow[t]{3}{*}{ 4-6 days/week } & Increase & 1.769 & $1.527-2.049$ & $<0.001$ \\
\hline & Decrease & 1.191 & $1.031-1.376$ & 0.018 \\
\hline & Uncertain & 1.154 & $0.834-1.595$ & 0.388 \\
\hline \multirow[t]{3}{*}{ 2-3 days/week } & Increase & 2.635 & $2.236-3.166$ & $<0.001$ \\
\hline & Decrease & 1.486 & $1.293-1.707$ & $<0.001$ \\
\hline & Uncertain & 1.123 & $0.869-1.451$ & 0.377 \\
\hline \multirow[t]{3}{*}{$\leq 1$ day/week } & Increase & 1.957 & $1.502-2.548$ & $<0.001$ \\
\hline & Decrease & 1.499 & $1.328-1.692$ & $<0.001$ \\
\hline & Uncertain & 1.002 & $0.806-1.246$ & 0.984 \\
\hline \multirow[t]{3}{*}{ Total } & Increase & 2.115 & $1.953-2.290$ & $<0.001$ \\
\hline & Decrease & 1.246 & $1.167-1.330$ & $<0.001$ \\
\hline & Uncertain & 0.881 & $0.768-1.010$ & 0.069 \\
\hline \multicolumn{5}{|c|}{ Intake frequency of sugary foods } \\
\hline \multirow[t]{3}{*}{ Every day } & Increase & 1.683 & $1.424-1.988$ & $<0.001$ \\
\hline & Decrease & 1.049 & $0.902-1.219$ & 0.534 \\
\hline & Uncertain & 0.944 & $0.663-1.345$ & 0.751 \\
\hline \multirow[t]{3}{*}{ 4-6 days/week } & Increase & 2.273 & $1.974-2.617$ & $<0.001$ \\
\hline & Decrease & 1.446 & $1.259-1.661$ & $<0.001$ \\
\hline & Uncertain & 0.931 & $0.699-1.239$ & 0.623 \\
\hline \multirow[t]{2}{*}{ 2-3 days/week } & Increase & 2.332 & $2.009-2.708$ & $<0.001$ \\
\hline & Decrease & 1.437 & $1.276-1.618$ & $<0.001$ \\
\hline
\end{tabular}


Table 5 Multivariate analysis of associations between frontline epidemic prevention experience and willingness to change dietary habits after experiencing the outbreak of COVID-19 (Continued)

\begin{tabular}{|c|c|c|c|c|}
\hline Intake frequency of dietary habits ${ }^{a}$ & Willingness $^{\mathrm{b}}$ & OR & OR $(95 \% \mathrm{Cl})$ & $P$-value \\
\hline & Uncertain & 1.160 & $0.937-1.437$ & 0.172 \\
\hline \multirow[t]{3}{*}{$\leq 1$ day/week } & Increase & 2.562 & $1.896-3.462$ & $<0.001$ \\
\hline & Decrease & 1.553 & $1.307-1.845$ & $<0.001$ \\
\hline & Uncertain & 1.028 & $0.801-1.319$ & 0.830 \\
\hline \multirow[t]{3}{*}{ Total } & Increase & 2.134 & $1.967-2.304$ & $<0.001$ \\
\hline & Decrease & 1.269 & $1.189-1.355$ & $<0.001$ \\
\hline & Uncertain & 0.879 & $0.775-0.996$ & 0.043 \\
\hline \multicolumn{5}{|l|}{ Intake frequency of pickled vegetables } \\
\hline \multirow[t]{3}{*}{ Every day } & Increase & 1.526 & $1.244-1.873$ & $<0.001$ \\
\hline & Decrease & 1.073 & $0.888-1.297$ & 0.465 \\
\hline & Uncertain & 0.946 & $0.621-1.441$ & 0.795 \\
\hline \multirow[t]{3}{*}{ 4-6 days/week } & Increase & 1.730 & $1.489-2.010$ & $<0.001$ \\
\hline & Decrease & 1.193 & $1.027-1.386$ & 0.021 \\
\hline & Uncertain & 0.940 & $0.680-1.300$ & 0.709 \\
\hline \multirow[t]{3}{*}{ 2-3 days/week } & Increase & 1.869 & $1.597-2.188$ & $<0.001$ \\
\hline & Decrease & 1.388 & $1.213-1.587$ & $<0.001$ \\
\hline & Uncertain & 1.180 & $0.929-1.497$ & 0.175 \\
\hline \multirow[t]{3}{*}{$\leq 1$ day/week } & Increase & 2.451 & $1.901-3.159$ & $<0.001$ \\
\hline & Decrease & 1.601 & $1.416-1.811$ & $<0.001$ \\
\hline & Uncertain & 1.072 & $0.887-1.297$ & 0.470 \\
\hline \multirow[t]{3}{*}{ Total } & Increase & 2.026 & $1.873-2.191$ & $<0.001$ \\
\hline & Decrease & 1.325 & $1.241-1.415$ & $<0.001$ \\
\hline & Uncertain & 0.906 & $0.801-1.024$ & 0.114 \\
\hline \multicolumn{5}{|c|}{ Intake frequency of processed meat products } \\
\hline \multirow[t]{3}{*}{ Every day } & Increase & 1.505 & $1.241-1.826$ & $<0.001$ \\
\hline & Decrease & 1.170 & $0.973-1.407$ & 0.095 \\
\hline & Uncertain & 0.759 & $0.478-1.207$ & 0.244 \\
\hline \multirow[t]{3}{*}{ 4-6 days/week } & Increase & 1.634 & $1.423-1.876$ & $<0.001$ \\
\hline & Decrease & 1.095 & $0.953-1.259$ & 0.200 \\
\hline & Uncertain & 0.914 & $0.686-1.219$ & 0.542 \\
\hline \multirow[t]{3}{*}{ 2-3 days/week } & Increase & 2.000 & $1.731-2.311$ & $<0.001$ \\
\hline & Decrease & 1.402 & $1.240-1.584$ & $<0.001$ \\
\hline & Uncertain & 1.032 & $0.825-1.291$ & 0.784 \\
\hline \multirow[t]{3}{*}{$\leq 1$ day/week } & Increase & 1.616 & $1.233-2.117$ & 0.001 \\
\hline & Decrease & 1.577 & $1.371-1.813$ & $<0.001$ \\
\hline & Uncertain & 1.176 & $0.962-1.439$ & 0.114 \\
\hline \multirow[t]{3}{*}{ Total } & Increase & 1.885 & $1.747-2.034$ & $<0.001$ \\
\hline & Decrease & 1.234 & $1.156-1.318$ & $<0.001$ \\
\hline & Uncertain & 0.855 & $0.756-0.956$ & 0.011 \\
\hline
\end{tabular}

${ }^{a}$ Baseline intake frequency of dietary habits was regarded as a stratification factor

bUnwillingness to change dietary habits was regarded as a reference group

'Frontline epidemic prevention experience was classified into dichotomous variables in the models (regarding "have the frontline epidemic prevention experience" as 1, and "no frontline epidemic prevention experience" as 2), adjusting for other characteristic factors (gender, age, BMl, education level marital status, occupation, and main living place) 
Table 6 Multivariate analysis of associations between frontline epidemic prevention experience and willingness to change behavioral habits after experiencing the outbreak of COVID-19

\begin{tabular}{|c|c|c|c|c|}
\hline Intake frequency of dietary habits ${ }^{a}$ & Willingness $^{\mathrm{b}}$ & OR & OR $(95 \% \mathrm{Cl})$ & $P$-value \\
\hline \multicolumn{5}{|l|}{ Smoking cigarettes/day } \\
\hline \multirow[t]{4}{*}{$>20$ cigarettes/day } & Increase & 2.048 & $0.869-4.831$ & 0.101 \\
\hline & Decrease & 1.547 & $0.717-3.342$ & 0.266 \\
\hline & Quit smoking & 3.054 & $1.277-7.308$ & 0.012 \\
\hline & Uncertain & 1.031 & $0.423-2.516$ & 0.946 \\
\hline \multirow[t]{4}{*}{ 11-20 cigarettes/day } & Increase & 2.067 & $1.359-3.143$ & 0.001 \\
\hline & Decrease & 1.659 & $1.152-2.389$ & 0.007 \\
\hline & Quit smoking & 1.885 & $1.263-2.813$ & 0.002 \\
\hline & Uncertain & $1 . .740$ & $0.967-3.131$ & 0.064 \\
\hline \multirow[t]{4}{*}{ 6-10 cigarettes/day } & Increase & 1.799 & $1.392-2.324$ & $<0.001$ \\
\hline & Decrease & 1.375 & $1.081-1.749$ & 0.010 \\
\hline & Quit smoking & 1.199 & $0.903-1.592$ & 0.210 \\
\hline & Uncertain & 0.817 & $0.466-1.433$ & 0.481 \\
\hline \multirow[t]{4}{*}{ 1-5 cigarettes/day } & Increase & 1.645 & $1.340-2.019$ & $<0.001$ \\
\hline & Decrease & 1.191 & $0.983-1.443$ & 0.074 \\
\hline & Quit smoking & 1.097 & $0.862-1.395$ & 0.452 \\
\hline & Uncertain & 0.757 & $0.478-1.201$ & 0.237 \\
\hline \multirow[t]{4}{*}{ No smoking } & Increase & 2.126 & $1.712-2.639$ & $<0.001$ \\
\hline & Decrease & 1.349 & $1.154-1.577$ & $<0.001$ \\
\hline & Quit smoking & 1.370 & $1.206-1.557$ & $<0.001$ \\
\hline & Uncertain & 0.825 & $0.642-1.061$ & 0.134 \\
\hline \multirow[t]{4}{*}{ Total } & Increase & 2.414 & $2.212-2.635$ & $<0.001$ \\
\hline & Decrease & 1.735 & $1.617-1.861$ & $<0.001$ \\
\hline & Quit smoking & 2.239 & $1.825-2.747$ & $<0.001$ \\
\hline & Uncertain & 1.006 & $0.842-1.202$ & 0.950 \\
\hline \multicolumn{5}{|l|}{ Alcohol consumption/week } \\
\hline \multirow[t]{4}{*}{ Every day } & Increase & 1.422 & $1.085-1.865$ & 0.011 \\
\hline & Decrease & 1.068 & $0.831-1.372$ & 0.609 \\
\hline & Quit drinking & 1.324 & $0.855-1.981$ & 0.172 \\
\hline & Uncertain & 1.022 & $0.502-2.080$ & 0.953 \\
\hline \multirow[t]{4}{*}{ 4-6 days/week } & Increase & 1.206 & $0.972-1.495$ & 0.089 \\
\hline & Decrease & 0.975 & $0.788-1.206$ & 0.815 \\
\hline & Quit drinking & 1.228 & $0.920-1.639$ & 0.163 \\
\hline & Uncertain & 0.313 & $0.163-0.599$ & $<0.001$ \\
\hline \multirow[t]{4}{*}{ 2-3 days/week } & Increase & 1.814 & $1.460-2.255$ & $<0.001$ \\
\hline & Decrease & 1.448 & $1.196-1.754$ & $<0.001$ \\
\hline & Quit drinking & 1.448 & $1.146-1.831$ & 0.002 \\
\hline & Uncertain & 0.788 & $0.485-1.281$ & 0.337 \\
\hline \multirow[t]{4}{*}{$\leq 1$ day/week } & Increase & 1.723 & $1.330-2.230$ & $<0.001$ \\
\hline & Decrease & 1.379 & $1.166-1.632$ & $<0.001$ \\
\hline & Quit drinking & 1.447 & $1.175-1.781$ & $<0.001$ \\
\hline & Uncertain & 1.048 & $0.754-1.458$ & 0.779 \\
\hline No drinking & Increase & 1.389 & $0.877-2.200$ & 0.161 \\
\hline
\end{tabular}


Table 6 Multivariate analysis of associations between frontline epidemic prevention experience and willingness to change behavioral habits after experiencing the outbreak of COVID-19 (Continued)

\begin{tabular}{|c|c|c|c|c|}
\hline Intake frequency of dietary habits ${ }^{a}$ & Willingness $^{\mathrm{b}}$ & OR & OR $(95 \% \mathrm{Cl})$ & $P$-value \\
\hline & Decrease & 1.327 & $1.078-1.632$ & 0.008 \\
\hline & Quit drinking & 1.321 & $1.140-1.530$ & $<0.001$ \\
\hline & Uncertain & 0.987 & $0.808-1.206$ & 0.898 \\
\hline \multirow[t]{4}{*}{ Total } & Increase & 2.320 & $2.126-2.530$ & $<0.001$ \\
\hline & Decrease & 1.720 & $1.608-1.840$ & $<0.001$ \\
\hline & Quit drinking & 1.536 & $1.406-1.679$ & $<0.001$ \\
\hline & Uncertain & 0.900 & $0.775-1.045$ & 0.166 \\
\hline \multicolumn{5}{|c|}{ Sleep duration/day (including lunch break) } \\
\hline \multirow[t]{3}{*}{$<5$ h/day } & Increase & 1.098 & $0.874-1.379$ & 0.423 \\
\hline & Decrease & 1.134 & $0.819-1.569$ & 0.449 \\
\hline & Uncertain & 0.549 & $0.320-0.942$ & 0.030 \\
\hline \multirow[t]{3}{*}{ 5-6 h/day } & Increase & 1.442 & $1.185-1.754$ & $<0.001$ \\
\hline & Decrease & 1.634 & $1.301-2.053$ & $<0.001$ \\
\hline & Uncertain & 0.858 & $0.589-1.249$ & 0.424 \\
\hline \multirow[t]{3}{*}{ 6-7 h/day } & Increase & 1.596 & $1.411-1.806$ & $<0.001$ \\
\hline & Decrease & 2.149 & $1.856-2.488$ & $<0.001$ \\
\hline & Uncertain & 1.193 & $0.925-1.539$ & 0.174 \\
\hline \multirow[t]{3}{*}{ 7-8 h/day } & Increase & 1.334 & $1.197-1.486$ & $<0.001$ \\
\hline & Decrease & 1.798 & $1.548-2.089$ & $<0.001$ \\
\hline & Uncertain & 1.192 & $0.965-1.472$ & 0.103 \\
\hline \multirow[t]{3}{*}{$>8$ h/day } & Increase & 2.065 & $1.689-2.525$ & $<0.001$ \\
\hline & Decrease & 1.279 & $1.016-1.610$ & 0.036 \\
\hline & Uncertain & 1.282 & $0.969-1.697$ & 0.082 \\
\hline \multirow[t]{3}{*}{ Total } & Increase & 1.491 & $1.401-1.587$ & $<0.001$ \\
\hline & Decrease & 1.736 & $1.603-1.880$ & $<0.001$ \\
\hline & Uncertain & 1.057 & $0.932-1.199$ & 0.388 \\
\hline \multicolumn{5}{|l|}{ Physical exercise time/week } \\
\hline \multirow[t]{3}{*}{$<30 \mathrm{~min} /$ week } & Increase & 0.759 & $0.667-0.863$ & $<0.001$ \\
\hline & Decrease & 1.379 & $1.041-1.828$ & 0.025 \\
\hline & Uncertain & 0.447 & $0.311-0.641$ & $<0.001$ \\
\hline \multirow[t]{3}{*}{30 min-1 h/week } & Increase & 1.279 & $1.153-1.419$ & $<0.001$ \\
\hline & Decrease & 1.495 & $1.265-1.766$ & $<0.001$ \\
\hline & Uncertain & 0.753 & $0.543-1.044$ & 0.088 \\
\hline \multirow[t]{3}{*}{ 1-2 h/week } & Increase & 1.397 & $1.210-1.613$ & $<0.001$ \\
\hline & Decrease & 1.641 & $1.381-1.950$ & $<0.001$ \\
\hline & Uncertain & 0.940 & $0.661-1.336$ & 0.730 \\
\hline \multirow[t]{3}{*}{ 2-3 h/week } & Increase & $1.1-9$ & $0.868-1.417$ & 0.407 \\
\hline & Decrease & 1.176 & $0.900-1.536$ & 0.236 \\
\hline & Uncertain & 0.982 & $0.644-1.496$ & 0.931 \\
\hline \multirow[t]{3}{*}{$>3$ h/week } & Increase & 1.162 & $0.862-1.566$ & 0.326 \\
\hline & Decrease & 1.123 & $0.753-1.675$ & 0.569 \\
\hline & Uncertain & 1.312 & $0.819-2.103$ & 0.259 \\
\hline Total & Increase & 1.270 & $1.191-1.117$ & $<0.001$ \\
\hline
\end{tabular}


Table 6 Multivariate analysis of associations between frontline epidemic prevention experience and willingness to change behavioral habits after experiencing the outbreak of COVID-19 (Continued)

\begin{tabular}{lllll}
\hline Intake frequency of dietary habits $^{\mathbf{a}}$ & Willingness $^{\mathbf{b}}$ & OR & OR (95\% Cl) & $\boldsymbol{P}_{\text {-value }}$ \\
\hline & Decrease & 1.588 & $1.451-1.739$ & $<0.001$ \\
& Uncertain & 0.828 & $0.705-0.971$ & 0.020 \\
\hline
\end{tabular}

Baseline intake frequency of behavioral habits was regarded as a stratification factor

${ }^{b}$ Unwillingness to change behavior habits was regarded as a reference group

'Frontline epidemic prevention experience was classified into dichotomous variables in the models (regarding "have the frontline epidemic prevention experience" as 1, and "no frontline epidemic prevention experience" as 2), adjusting for other characteristic factors (gender, age, BMl, education level marital status, occupation, and main living place)

Chew et al. [16, 17] found that regardless of the volume of cases or deaths, health care workers from all five Asian-Pacific countries were vulnerable to psychological distress during the outbreak of COVID-19, and Tan et al. [18] reported that the prevalence of psychological adversity was higher among nonmedical health care workers than among health care workers during the COVID-19 outbreak in Singapore. Chew et al. [17] also found that psychological disorders were significantly associated with physical symptoms. Le et al. and Le et al. $[29,30]$ reported that depression and anxiety were associated with being in contact with potential COVID-19 patients. Therefore, psychological interventions are urgent for protecting frontline epidemic prevention workers from diverse psychological outcomes during COVID-19.

In addition to a negative effect on psychology, COVID-19 also has a negative impact on dietary and behavioral habits. The pandemic has enforced numerous restrictions on daily living including social distancing, isolation, and home confinement [4]. These measures may have caused many negative changes in lifestyle behaviors, including eating more unhealthy diets, engaging in less physical activity, smoking more cigarettes and consuming more alcohol, and increases in depression and anxiety, which makes individuals more susceptible to chronic diseases as well as COVID-19 [19, 29-31]. Husain et al. reported that COVID-19 changed dietary habits and lifestyle behaviors in Kuwait, including skipping breakfast, having more late-night snacks and daytime naps, and getting less physical activity and nighttime sleep [32]. Our previous study showed that high epidemic concern was positively correlated with poor dietary habits during the COVID-19 outbreak [33]. Our current study focused on frontline epidemic prevention workers who had frontline epidemic prevention experience during the epidemic outbreak in China. We aimed to identify their baseline dietary and behavioral habits before the COVID-19 period and further explore their willingness to change dietary and behavioral habits after experiencing the outbreak of COVID-19 based on a cross-sectional study. To further explore targeted strategies, the susceptible subgroups were identified by stratified analyses for subsequent protection from being infected with COVID-19.

The average age of 9402 frontline epidemic prevention workers in this study was $28.09 \pm 7.50$ years old, which was younger than that of health care workers in five Asian-Pacific countries [16]. Our results found that almost all dietary and behavioral habits of frontline epidemic prevention workers were worse than those of other participants before the epidemic. Christensen et al. also reported that health care workers had a high prevalence of obesity and musculoskeletal pain and low physical capacities [34]. These conclusions might be ascribed to their poor lifestyles, night shifts, and other characteristics of their occupation.

After experiencing the outbreak of COVID-19, most frontline epidemic prevention workers tended to choose healthy dietary and behavioral habits. The numerical results showed that frontline epidemic prevention experience exerted a huge impact on dietary and behavioral habits, and frontline epidemic prevention workers expressed a greater willingness to adopt healthy dietary and behavioral habits than others. These meaningful changes in lifestyle behaviors during COVID-19 were consistent with what Balanzá-Martínez reported [35]; however, they also found that being an essential worker, having worse self-rated health, obtaining a positive screening for depression/anxiety, and making substantial changes in diet/nutrition and sleep were all associated with poorer lifestyles.

It is worth noting that some frontline epidemic prevention workers with bad dietary and behavioral habits before COVID-19 expressed a willingness to adopt worse habits after experiencing the outbreak of COVID19. As Di Renzo et al. reported, the epidemic clearly affected the dietary and behavioral habits of the Italian population during COVID-19 [27]. Healthy dietary and behavioral habits could boost immunity, prevent or treat chronic diseases, including hypertension, diabetes, and insomnia [26, 36, 37] and prolong life [38]. People with bad dietary and behavioral habits or chronic diseases have been more susceptible to COVID-19 [21]. Ammar reported that isolation was a necessary measure to protect public health, but the results indicated that isolation altered physical activity and eating behaviors in a health- 
compromising direction [19]. These results might be ascribed to boredom, anxiety, out of control eating, more snacking between meals, social distancing, and isolation at home during the COVID-19 period [39].

All the above findings suggest that dietary and behavioral intervention policies are urgently needed to protect frontline epidemic prevention workers, especially those that had poor dietary and behavioral habits before the COVID-19 outbreak. Internet-based cognitive behavioral therapy may be a good choice for treating frontline epidemic prevention workers with psychiatric symptoms and addressing unhealthy dietary and behavior lifestyles during the pandemic [40]. Online therapy, rather than face-to-face meetings, has the potential to reduce body dissatisfaction, anxiety, and depression during the COVID-19 period [41-43]. It is necessary to establish and improve the working conditions for those in epidemic prevention work. In addition, receiving medical training is needed before fighting the epidemic. Another suggestion is that frontline epidemic prevention workers should be in good health without prior medical conditions [16].

We acknowledge certain limitations of the study. First, a cross-sectional study does not allow us to assess the causality between frontline epidemic prevention experience and willingness to change dietary and behavioral habits after experiencing the outbreak of COVID-19. Second, questionnaires were conducted without strict control measures. However, our study has several advantages, including a large sample size and a wide range of types of frontline epidemic prevention workers, including medical workers, community workers, policemen, volunteers, and others who took part in the fight against COVID-19. Moreover, our questionnaires were conducted after the outbreak of COVID-19 in China, while most of the previous questionnaires had been conducted during the outbreak of COVID-19. Therefore, our findings are helpful for protecting more frontline epidemic prevention workers after the initial outbreak of COVID19 by examining the adopting of healthy dietary and behavioral habits.

\section{Conclusions}

In summary, our conclusions were based on a large sample size, anonymity, and a confidential internet questionnaire. Our findings suggest that the dietary and behavioral habits of frontline epidemic prevention workers were worse than those of other participants before the COVID-19 outbreak. The frontline epidemic prevention experience exerted a huge impact on their dietary and behavioral habits, and frontline epidemic prevention workers had the willingness to adopt healthy dietary and behavioral habits after experiencing the outbreak of COVID-19. Some frontline epidemic prevention workers with poor dietary and behavioral habits before the COVID-19 outbreak expressed a willingness to engage in worse habits after experiencing the outbreak of COVID-19. Therefore, dietary and behavioral intervention policies are urgently needed to protect their health, especially for those who have poor dietary and behavioral habits.

\section{Abbreviations}

COVID-19: 2019 Novel coronavirus disease; WHO: World health Organization; BMl: Body mass index

\section{Acknowledgements}

We really appreciate all the participants in this study, and thanks for the support by all the team members for this study.

\section{Authors' contributions}

$W Y$ and QG designed the whole process and were the main drafters of the manuscript. ZL, XH, YM, FC, ZL, RZ, and DW did some of the expeditionary research and contributed the coordination and manuscript editing. QY, YG, and JC were the directors for the fund and designed ideas of research. All participated in the analysis and discussion under the leadership and instruction of JZ, JC, and YX. All authors read and approved the final manuscript.

\section{Funding}

This work was supported by The Science and Technology Project of Bao'an (no. 2020JD101)

\section{Availability of data and materials}

All data in the study can be accessed from the corresponding author upon request.

\section{Declaration}

\section{Ethics approval and consent to participate}

This study was approved by the ethics committee of Bao'an District Hospital for Chronic Diseases Prevention and Cure. Informed consent was obtained from all subjects or, if subjects are under 18, from a parent and/or legal guardian. All methods were carried out in accordance with relevant guidelines and regulations.

\section{Consent for publication}

Not applicable.

\section{Competing interests}

The authors declare that they have no competing interests.

\section{Author details}

'Bao'an District Hospital for Chronic Diseases Prevention and Cure, Shenzhen 518100, China. ${ }^{2}$ Shenzhen Hospital, Southern Medical University, Shenzhen 518101, China. ${ }^{3}$ School of Public Health, Guangdong Medical University, Dongguan 523808, China.

Received: 11 January 2021 Accepted: 28 April 2021

Published online: 18 May 2021

\section{References}

1. WHO. Coronavirus disease (COVID-19) Media resources: WHO DirectorGeneral's statement on IHR Emergency Committee on Novel Coronavirus (2019-nCoV). 2020. https://www.who.int/director-general/speeches/detail/ who-director-general-s-statement-on-ihr-emergency-committee-on-novelcoronavirus-(2019-ncov). (accessed December 31, 2020).

2. WHO. Coronavirus disease (COVID-19) Situation reports: WHO coronavirus disease (COVID-19) dashboard. 2020. https://covid19.who.int/ (accessed December 31, 2020)

3. Bong C, Brasher C, Chikumba E, McDougall R, Mellin-Olsen J, Enright A. The COVID-19 pandemic: effects on low- and middle-income countries. Anesth Analg. 2020;131(1):86-92. https://doi.org/10.1213/ANE.0000000000004846. 
4. $\quad$ Feng $W$, Zong W, Wang F, Ju S. Severe acute respiratory syndrome coronavirus 2 (SARS-CoV-2): a review. Mol Cancer. 2020;19(1):100. https://doi. org/10.1186/s12943-020-01218-1.

5. Wang C, Pan R, Wan X, Tan Y, Xu L, Ho CS, et al. Immediate psychological responses and associated factors during the initial stage of the 2019 coronavirus disease (COVID-19) epidemic among the general population in China. Int J Env Res Pub He. 2020;17(5):1729. (PMID: 32155789. https://doi. org/10.3390/ijerph17051729.

6. Nicola M, Alsafi Z, Sohrabi C, Kerwan A, Al-Jabir A, losifidis C, et al. The socio-economic implications of the coronavirus pandemic (COVID-19): a review. Int J Surg. 2020;78:185-93. (PMID: 32305533. https://doi.org/10.1016/ j.ijsu.2020.04.018

7. WHO. Coronavirus disease (COVID-19) media resources: WHO DirectorGeneral's opening remarks at the media briefing on COVID-19. 2020. https:// www.who.int/director-general/speeches/detail/who-director-general-sopening-remarks-at-the-media-briefing-on-covid-19\%2D\%2D-2-october-202 0. (accessed December 31, 2020).

8. Xu S, Li Y. Beware of the second wave of COVID-19. Lancet. 2020; 395(10233):1321-2. (PMID: 32277876. https://doi.org/10.1016/S0140-6736(2 0) $30845-X$

9. Wise J. Covid-19: Risk of second wave is very real, say researchers. BMJ. 2020;369:m2294. (PMID: 32518177. https://doi.org/10.1136/bmj.m2294.

10. Nagesh S, Chakraborty S. Saving the frontline health workforce amidst the COVID-19 crisis: challenges and recommendations. J Glob Health. 2020;10(1): 10345. (PMID: 32373323. https://doi.org/10.7189/jogh-10-010345.

11. Green A. Li Wenliang. Lancet 2020;395(10225):682. (DOI: https://doi.org/ https://doi.org/10.1016/50140-6736(20)30382-2)

12. The L. COVID-19: protecting health-care workers. Lancet (London, England). 2020;395(10228):922. (PMID: 32199474. https://doi.org/10.1016/S0140-6736(2 0)30644-9.

13. Li Z, Ge J, Yang M, Feng J, Qiao M, Jiang R, et al. Vicarious traumatization in the general public, members, and non-members of medical teams aiding in COVID-19 control. Brain Behav Immun. 2020;88:916-9. (PMID: 32169498. https://doi.org/10.1016/j.bbi.2020.03.007

14. Sim MR. The COVID-19 pandemic: major risks to healthcare and other workers on the front line. Occup Environ Med. 2020;77(5):281-2. (PMID: 32238444. https://doi.org/10.1136/oemed-2020-106567.

15. Wang C, Pan R, Wan X, Tan Y, Xu L, Ho CS, et al. Immediate psychological responses and associated factors during the initial stage of the 2019 coronavirus disease (COVID-19) epidemic among the general population in China. Int J Env Res Pub He. 2020;17(5):1729. (PMID: 32155789. https://doi. org/10.3390/ijerph17051729.

16. Chew NWS, Ngiam JN, Tan BY, Tham S, Tan CY, Jing M, et al. Asian-Pacific perspective on the psychological well-being of healthcare workers during the evolution of the COVID-19 pandemic. BJPsych Open. 2020;6(6):e116. https://doi.org/10.1192/bjo.2020.98

17. Chew NWS, Lee GKH, Tan BYQ, Jing M, Goh Y, Ngiam NJH, et al. A multinational, multicentre study on the psychological outcomes and associated physical symptoms amongst healthcare workers during COVID19 outbreak. Brain Behav Immunity. 2020;88:559-65. https://doi.org/10.1016/ j.bbi.2020.04.049.

18. Tan B, Chew N, Lee G, Jing M, Goh Y, Yeo L, et al. Psychological impact of the COVID-19 pandemic on health care workers in Singapore. Ann Intern Med. 2020;173(4):317-20. https://doi.org/10.7326/M20-1083.

19. Ammar A, Brach M, Trabelsi K, Chtourou H, Boukhris O, Masmoudi L, et al. Effects of COVID-19 home confinement on eating behaviour and physical activity: results of the ECLB-COVID19 International online survey. Nutrients. 2020;12(6):1583. (PMID: 32481594. https://doi.org/10.33 90/nu12061583

20. Willett WC, Koplan JP, Nugent R, Dusenbury C, Puska P, Gaziano TA. Prevention of chronic disease by means of diet and lifestyle changes. [Review; Book Chapter]. 2006 2006-01-20.

21. Li X, Xu S, Yu M, Wang K, Tao Y, Zhou Y, et al. Risk factors for severity and mortality in adult COVID-19 inpatients in Wuhan. J Allergy Clin Immunol. 2020; 146(1):110-8. (PMID: 32294485. https://doi.org/10.1016/j.jaci.2020.04.006.

22. Halpin DMG, Faner $R$, Sibila O, Badia JR, Agusti A. Do chronic respiratory diseases or their treatment affect the risk of SARS-CoV-2 infection? Lancet Respir Med. 2020;8(5):436-8. (PMID: 32251625. https://doi.org/10.1016/ S2213-2600(20)30167-3

23. Côté $A$, Ternacle J, Pibarot $P$. Early prediction of the risk of severe coronavirus disease 2019: a key step in therapeutic decision making.
Ebiomedicine. 2020;59:102948. (PMID: 32810827. https://doi.org/10.1016/j. ebiom.2020.102948.

24. Xiao L, Zhang W, Gong M, Zhang Y, Chen L, Zhu H, et al. Development and validation of the HNC-LL score for predicting the severity of coronavirus disease 2019. Ebiomedicine. 2020;57:102880. (PMID: 32645614. https://doi. org/10.1016/j.ebiom.2020.102880.

25. Johnson TP. Snowball Sampling. In: Encyclopedia of Biostatistics. Wiley; 2005. https://doi.org/10.1002/0470011815.b2a16070.

26. Block G, Azar KMJ, Romanelli RJ, Block TJ, Palaniappan LP, Dolginsky M, et al. Improving diet, activity and wellness in adults at risk of diabetes: randomized controlled trial. Nutr Diabetes. 2016;6(9):e231. (PMID: 27643726 https://doi.org/10.1038/nutd.2016.42.

27. Zhou F, Yu T, Du R, Fan G, Liu Y, Liu Z, et al. Clinical course and risk factors for mortality of adult inpatients with COVID-19 in Wuhan, China: a retrospective cohort study. Lancet. 2020;395(10229):1054-62.

28. Remuzzi A, Remuzzi G. COVID-19 and Italy: what next? Lancet. [Journal Article; Review]. 2020;395:1225-1228. (PMID: 32178769, DOl: https://doi.org/1 0.1016/S0140-6736(20)30627-9)

29. Le HT, Lai AJX, Sun J, Hoang MT, Vu LG, Pham HQ, et al. Anxiety and depression among people under the nationwide partial lockdown in Vietnam. Front Public Health. 2020;8. https://doi.org/10.3389/fpubh.2020.589359.

30. Le XTT, Dang AK, Toweh J, Nguyen QN, Le HT, Do TTT, et al. Evaluating the psychological impacts related to COVID-19 of Vietnamese people under the first nationwide partial lockdown in Vietnam. Front Psychiatry. 2020;11:824. https://doi.org/10.3389/fpsyt.2020.00824.

31. Di Renzo L, Gualtieri P, Pivari F, Soldati L, Attinà A, Cinelli G, et al. Eating habits and lifestyle changes during COVID-19 lockdown: an Italian survey. J Transl Med. 2020;18(1):229. https://doi.org/10.1186/s12967-020-02399-5.

32. Husain W, Ashkanani F. Does COVID-19 change dietary habits and lifestyle behaviours in Kuwait: a community-based cross-sectional study. Environ Health Prev Med. 2020;25(1):61. (PMID: 33045996. https://doi.org/10.1186/ s12199-020-00901-5.

33. Xu Y, Li Z, Yu W, He X, Ma Y, Cai F, et al. The association between subjective impact and the willingness to adopt healthy dietary habits after experiencing the outbreak of the 2019 novel coronavirus disease (COVID19): a cross-sectional study in China. Aging (Albany NY). 2020;12(21):2096881. https://doi.org/10.18632/aging.103929.

34. Christensen JR, Faber A, Ekner D, Overgaard K, Holtermann A, Sogaard K. Diet, physical exercise and cognitive behavioral training as a combined workplace based intervention to reduce body weight and increase physical capacity in health care workers - a randomized controlled trial. Bmc Public Health. [Journal Article; Randomized Controlled Trial; Research Support, Non-U.S. Gov't]. 2011; 11:671. (PMID: 21871113, DOI: https://doi.org/10.11 86/1471-2458-11-671)

35. Balanzá-Martínez V, Kapczinski F, de Azevedo CT, Atienza-Carbonell B, Rosa AR, Mota JC, et al. The assessment of lifestyle changes during the COVID-19 pandemic using a multidimensional scale. Rev Psiquiatr Salud. 2020:S18889891. (PMID: 32962948. https://doi.org/10.1016/j.rpsm.2020.07.003.

36. Akahane T, Namisaki T, Kaji K, Moriya K, Kawaratani H, Takaya H, et al. Chronic alcohol consumption is inversely associated with insulin resistance and fatty liver in Japanese males. Nutrients. 2020;12(4):1036. (PMID: 32283773. https://doi.org/10.3390/nu12041036.

37. Murai U, Yamagishi K, Sata M, Kokubo Y, Saito I, Yatsuya H, et al. Seaweed intake and risk of cardiovascular disease: the Japan Public Health Centerbased Prospective (JPHC) Study. Am J Clin Nutr. 2019:110(6):1449-55. https://doi.org/10.1093/ajcn/nqz231

38. Li Y, Pan A, Wang DD, Liu X, Dhana K, Franco OH, et al. Impact of healthy lifestyle factors on life expectancies in the US population. Circulation. 2018; 138(4):345-55. (PMID: 29712712. https://doi.org/10.1161/CIRCULATIONA HA.117.032047.

39. Moynihan AB, van Tilburg WAP, Igou ER, Wisman A, Donnelly AE, Mulcaire JB. Eaten up by boredom: consuming food to escape awareness of the bored self.; 2015; 6:. 369. (PMID: 25883579, DOI: https://doi.org/10.3389/ fpsyg.2015.00369)

40. Ho CS, Chee CY, Ho RC. Mental health strategies to combat the psychological impact of COVID-19 beyond paranoia and panic. Ann Acad Med Singap. 2020;49(3):155-60. https://doi.org/10.47102/annals-aca dmedsg.202043.

41. Zhang MWB, Ho RCM. Moodle: The cost effective solution for internet cognitive behavioral therapy (I-CBT) interventions. Technol Health Care. 2017;25(1):163-5. https://doi.org/10.3233/THC-161261. 
42. Soh HL, Ho RC, Ho CS, Tam WW. Efficacy of digital cognitive behavioural therapy for insomnia: a meta-analysis of randomised controlled trials. Sleep Med. 2020;75:315-25. https://doi.org/10.1016/j. sleep.2020.08.020.

43. Low TL, Ho R, Ho C, Tam W. The efficacy of virtual reality in the treatment of binge-purging eating disorders: A meta-analysis. Eur Eat Disord Rev. 2021; 29(1):52-9. https://doi.org/10.1002/erv.2804.

\section{Publisher's Note}

Springer Nature remains neutral with regard to jurisdictional claims in published maps and institutional affiliations.

Ready to submit your research? Choose BMC and benefit from:

- fast, convenient online submission

- thorough peer review by experienced researchers in your field

- rapid publication on acceptance

- support for research data, including large and complex data types

- gold Open Access which fosters wider collaboration and increased citations

- maximum visibility for your research: over $100 \mathrm{M}$ website views per year

At $\mathrm{BMC}$, research is always in progress.

Learn more biomedcentral.com/submissions 